\title{
Fast Exfoliation and Functionalisation of 2D Crystalline Carbon Nitride by Framework Charging
}

\author{
Jingjing Jia, Edward R. White, Adam J. Clancy, Noelia Rubio, Theo Suter, Thomas S. Miller, Paul F. \\ McMillan, Veronika Brázdová, Furio Corà, Chris A. Howard, Robert V. Law, Cecilia Mattevi, ${ }^{*}$ and Milo \\ S. P. Shaffer*
}

Graphitic carbon nitride ( $\mathrm{gCN})$ has triggered tremendous interest due to its 2D structure, analogous to graphene, but with complementary characteristics. ${ }^{[1]}$ In particular, it offers semiconductivity with tunable band gap and optical $\mathrm{C}$ create empty sites within the layers. ${ }^{[3]}$ Monolayer/few layer carbon nitride nanosheets (FL-CNs) have been isolated as a new family of $2 \mathrm{D}$ layered materials, motivated by their unique photocatalytic activity. ${ }^{[4]}$ Several methods have been

[*] J. Jia, E. R. White, A. J. Clancy, N. Rubio, M. S. P. Shaffer

Dept. Chemistry, Imperial College London,

London, SW7 2AZ, UK.

E-mail: m.shaffer@imperial.ac.uk

C. Mattevi

Dept. Materials, Imperial College London,

London, SW7 2AZ, UK

E-mail: c.mattevi@imperial.ac.uk

T. Suter, T. S. Miller, P. F. McMillan,

V. Brázdová, F. Corà,

Dept. Chemistry, University College London,

London, WC1H OAJ, UK.

C. A. Howard

Dept. Physics and Astronomy, University College London,

London, WC1E 6BT, UK.

Electronic supplementary information (ESI) for this article is given via a link at the end of the document.
35 properties of nanomaterials, ${ }^{[9]}$ to date there has been little 36 direct covalent functionalisation of PTI.

37 This paper demonstrates a simple, one-pot exfoliation, 38 dissolution, and optional functionalisation of FL-PTI via 39 reduction. Reductive charging has been used previously to 40 dissolve a variety of $2 \mathrm{D}$ nanomaterials, ${ }^{[10]}$ via metal-ammonia 41 solutions and organic charge transfer agents (CTA). The use 42 of sodium naphthalide ( $\mathrm{NaNp}$ ) dissolved in N,N43 dimethylacetamide (DMAc) was recently found to be 44 especially effective for the dissolution and functionalisation of 45 single wall nanotubes in a single step. ${ }^{[14]}$ Here the 46 methodology is adapted to gCNs, specifically PTIs.

47 Successful exfoliation of PTI was achieved by framework 48 charging process (Fig. 1). Sodium is used as the electron 49 source to form naphthalide ions, which act as a CTA. DMAc is 50 an excellent room temperature solvent for naphthalene/ide and 51 anionic nanocarbons, and can be expected to be a good 52 solvent for FL-PTI. ${ }^{[11]} \mathrm{NaNp} / \mathrm{DMAc}$ solution has a characteristic 53 green color which simplifies reaction monitoring. NaNp/DMAc 54 solutions were added into dried PTI powders at controlled 55 stoichiometry. The reduction and exfoliation process was 56 observed by the color change from the initial brown PTI 57 suspension to dark green after addition of $\mathrm{NaNp} / \mathrm{DMAc}$, before 58 finally forming a brilliant orange dispersion of FL-PTI (FL-PTI $\left.{ }^{n-}\right)$ 59 within minutes (Fig. S1). After removing the remaining 60 insoluble PTI fragments by centrifugation $(5000 \mathrm{~g}, 30 \mathrm{~min}$, Fig. $61 \mathrm{~S} 2)$, a homogeneous golden FL-PTI ${ }^{\mathrm{n}-}$ solution with a 62 concentration up to $1.2 \mathrm{mg} \cdot \mathrm{mL}^{-1}$ was obtained (Fig. S1c), 63 which was stable under $\mathrm{N}_{2}$ for $>1$ year (Fig. S3a). Deposited 64 FL-PTIn- nanosheets display hexagonal geometry with a height 65 of $1-2 \mathrm{~nm}$ (Fig. S3), indicating that they comprise only a few 66 PTI layers, based on a $3.52 \AA$ layer thickness. ${ }^{[6]}$ The negative 67 charges from the naphthalide are likely to be rapidly 68 transferred to the PTI, due to the high reduction potential of 69 naphthalide (ca. $3.0 \mathrm{eV}$ vs SHE); ${ }^{[12]}$ accelerated by the small 70 size of the PTI platelets and the intrinsic pores. ${ }^{[13]}$ The partially 71 dissociated $\mathrm{Na}^{+}$counterions leave a net unscreened negative 72 charge on the $\mathrm{PTI}^{\mathrm{n}-}$ (Fig. S4a), leading to short range 73 Coulombic repulsions and hence exfoliation of PTI into 74 solvated FL-PTI $^{\text {n- }}$ sheets (Fig. 1), analogous to reduced 75 nanocarbons $^{[10 \mathrm{a}, 14]}$ and transition metal dichalcogenides. ${ }^{[10 \mathrm{~b}]}$ 76 Upon air exposure, the FL-PTI reagglomerate slowly ( 2 77 months, Fig. S5b); the reduced rate compared to charged 78 sWCNTs, ${ }^{[14 \mathrm{~b}]}$ likely relates to the lower aspect ratio and 79 localisation/low mobility of the charges on the framework. ${ }^{[15]}$ 

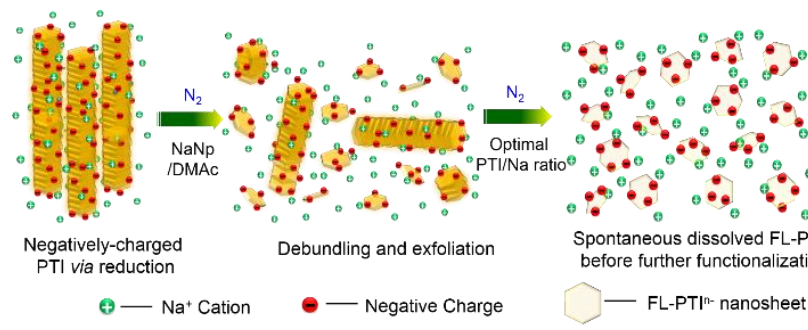

$\because \% 0.00$

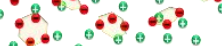
$\because \because \because \because \%$ Spontaneous dissolved FL-PT before further functionalization - FL-PTIn- nanosheet

Figure 1. Schematic of charging and exfoliation of PTI.

The charging ratio (molar [PTI framework atoms]:Na (ESI), 83 weighted $\mathrm{M}_{\mathrm{w}}(\mathrm{PTI})=13.14$ ) and initial PTI loading (mg PTI/ $\mathrm{mL}$ of $\mathrm{DMAc}$ ) are two vital factors affecting the exfoliation, controlling both the yield (mass fractions of PTI) and concentration of solubilised FL-PTIn-. Increasing the degree of charging (i.e. lower PTI:Na), at a static PTI loading initially led to an improved yield of FL-PTIn- $(4.1 \mathrm{wt} \%$ to $34.5 \mathrm{wt} \%)$ due to the enhanced Columbic repulsion (Fig. 2a). However, further increasing charge (PTI:Na $<7$ ) reduced the yield. Similar effects have been observed in charged nanocarbon solutions, attributed to $\mathrm{Na}^{+}$condensation and charge screening. ${ }^{[11,16]}$ The optimum $\mathrm{Na}$ concentration for exfoliation of PTI is $15 \mathrm{mM}$ (i.e. 7:1 PTI:Na for $1.4 \mathrm{mg} \cdot \mathrm{mL}^{-1}$, Fig. 2a), comparable to $\sim 10 \mathrm{mM}$ identified for the exfoliation of $\mathrm{Na}$ reduced graphite of similar geometry. ${ }^{[16]}$ At the highest charge regimes, the charge on the PTI saturates (at PTI:Na ratio of $\sim 5$ ), as observed by the green tinge of unreacted NaNp (Fig. 2a). On varying the initial PTI loading (Fig. 2b), the concentration of dissolved FL-PTI ${ }^{n-}$ scales linearly, giving a consistent yield between $31-35 \mathrm{wt} \%$, indicating that there may be an intrinsically soluble portion of the starting material. The residue, isolated after centrifugation may contain defects that bind the layers; indeed, qualitatively, the undissolved residue appears disordered by SEM (Fig. S2C). The maximum concentration of FL-PTIn- is $\sim 1.2 \mathrm{mg} \cdot \mathrm{mL}^{-1}$ from a PTI loading of $3.5 \mathrm{mg} \cdot \mathrm{mL}^{-1}$. Further increases in PTI loading did not increase the concentration, indicating the solution is saturated (Fig. 2b).
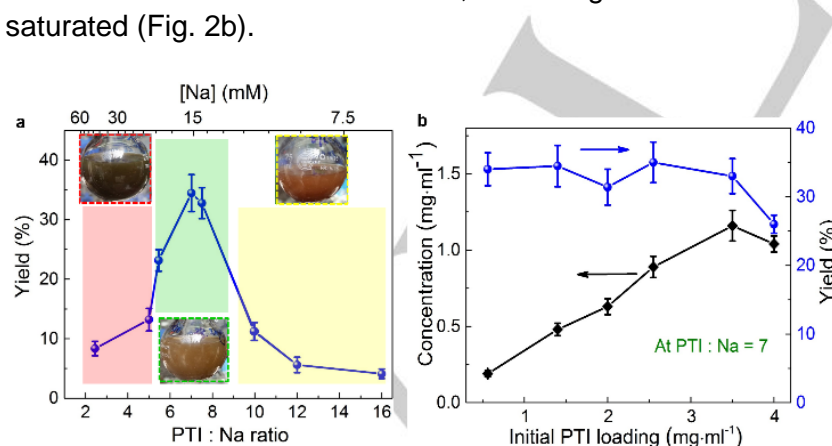

Figure 2. (a) FL-PTIn- yield versus PTI:Na ratio/[Na], $1.4 \mathrm{mg} \cdot \mathrm{mL}^{-1} \mathrm{PTI}$. Yellow, green and red rectangles correspond to low, to high $[\mathrm{Na}]$ respectively with inset photographs showing resultant FL-PTI ${ }^{\mathrm{n}}$ dispersions. (b) Effect of initial PTI loading on the concentration/yield of FL-PTI ${ }^{\text {n- }}$ dissolution (7:1 PTI:Na ratio).

117 Aqueous FL-PTI dispersions are desirable for 118 environmentally benign processing; however, pristine PTI
119 are poorly soluble in water due to their strong interlayer
120

121

122

123

124

125

126

127

128

129

130

131

132

133

134

135

136

137

138

139

140

141

142

143

144

145

146

147

148

149

150

151

152

153

154

155 interactions $(6 \mathrm{~h}$ probe sonication was found to give a concentration $<0.2 \mathrm{mg} \mathrm{mL}^{-1}$ ). The framework charging process overcomes the strong interlayer interactions and accelerates solubilisation in DMAc, allowing the removal of the intrinsically insoluble fraction of the pristine PTI. The FLPTIs can then be recovered from the DMAc by solvent exchange and the resultant FL-PTI can be transferred into water by solvent exchange, reaching a saturated concentration of $3.5 \mathrm{mg} \cdot \mathrm{mL}^{-1}$. Atomic force microscopy (AFM) confirms an excellent dispersion of FL-PTI in water (Fig. 3a), with corresponding heights of 0.33-3.2 nm (avg. $1.11 \mathrm{~nm}$, Fig. $3 \mathrm{~b}$ and S6), suggesting that the nanosheets mostly comprise $\sim 3$ PTI layers, although some monolayers are present. Representative energy dispersive X-ray spectroscopy (EDX) maps show uniform dispersion of $C$ and $\mathrm{N}$ throughout the whole hexagonal area of the exfoliated FLPTI nanosheet (Fig 3c-e). High-resolution transmission electron microscopy (HRTEM) micrographs show intact PTI crystallites with regular hexagonal geometry and clear facets (Fig. 4a). Notably, no defect holes or dislocations were observed, confirming the non-destructive nature of the framework charging exfoliation, as well as the high quality of the starting material. The fast Fourier transform (FFT) of the unfiltered HRTEM image shows a hexagonal lattice, demonstrating a single crystal exfoliated FL-PTI (Fig 4a and further examples in S9). ${ }^{[17]}$ The minimum reciprocal lattice vector, $G_{\text {min }}$, is $1.4 \mathrm{~nm}^{-1}$, giving a lattice constant $\boldsymbol{a}=(2 / \mathrm{V}$ 3) $G_{\min }=8.5 \AA$, consistent with the reported values from $\mathrm{Br}$ intercalated PTI. ${ }^{[15]}$ Two possible stacking models can be considered for the FL-PTI: AB stacking with aligned voids forming $C$-axis channels, and $A C$ stacking without channels in two adjacent layers (Fig. S10a). Comparing simulated electron diffraction patterns of these two models with the experimental selected area electron diffraction (SAED) data, the $A B$ stacking structure is the better fit for the FL-PTI nanosheets (Fig. 4b-d and S10b).
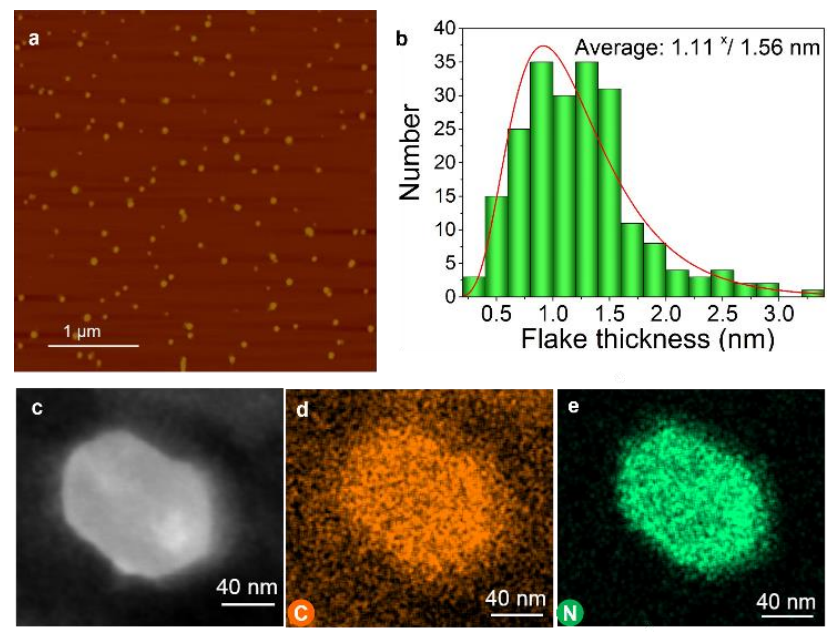

Figure 3. (a) AFM image of FL-PTI nanosheets. (b) PTI thickness histogram $(n>200)$. Mean value is derived from a lognormal distribution. (c) STEM image and EDX elemental maps of $C(d)$ and N (e) on a FL-PTI 
163
160

nanosheet. The background $\mathrm{C}$ signal in (d) is due to the carbon TEM support.

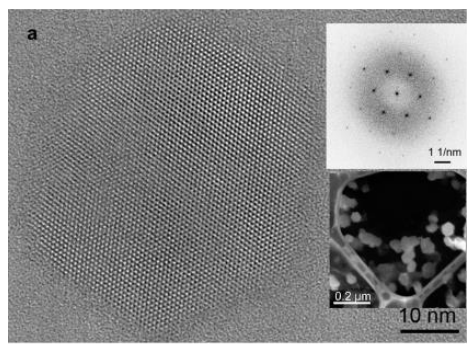

\section{b}

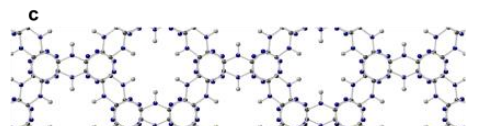

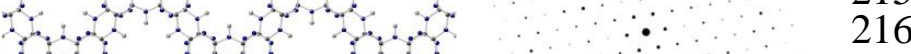

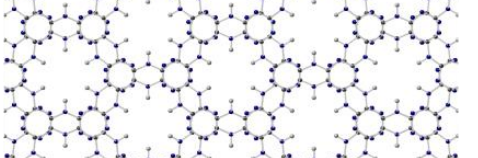

Figure 4. (a) HRTEM image of a FL-PTI nanosheet. Top inset: FFT of the HRTEM image, showing a single crystal hexagonal structure. Bottom inset: STEM image, indicating the layered structure of FL-PTI (b) SAED pattern of a FL-PTI nanosheet. (c) Schematic of the AB stacking in a bilayer. (d) Simulated electron diffraction pattern from the $A B$ crystal structure.

The FL-PTI ${ }^{\text {- }}$ synthesised in DMAc solution provides a versatile platform for covalent functionalisation of $\mathrm{PTI}$, comparable to negatively charged graphene and boron nitride nanotube counterparts. ${ }^{[16,18]}$ Pristine PTI and FL-PTI nanosheets are thermally stable up to $\sim 700$ and $670^{\circ} \mathrm{C}$, respectively (Fig. 5a and Figure S12); the slightly depressed decomposition temperature for FL-PTI reflects its fewlayered character. Functionalisation can be performed via simple addition of an alkyl halide to the reduced nanomaterial. After functionalisation by reaction with dodecyl bromide, a $10 \mathrm{wt} \%$ mass loss can be observed in thermogravimetric analysis/evolved-gas mass spectrometry (TGA-MS), relative to controls; the weight loss correlates with a $\mathrm{m} / \mathrm{z}$ peak at 57 , attributed to $\mathrm{C}_{4} \mathrm{H}_{9}{ }^{+}$from the grafted $\mathrm{C}_{12} \mathrm{H}_{25}$ alkyl chain (Fig. 5a). During the alkylation reaction, the solution changes from a clear golden, to a turbid pale yellow appearance; as the depletion of the negative charges progresses, the Coulombic stabilization is lost, leading to agglomeration and increased light scattering (Fig. 5b, S1c and S12). X-ray photoelectron spectroscopy (XPS) measurements confirmed the covalent attachment of alkyl chains to the PTI structure. Core level $\mathrm{N}$ 1s spectra can be divided into two components: $398.6 \mathrm{eV}$ corresponding to C$\mathrm{N}=\mathrm{C}$ groups and $400.9 \mathrm{eV}$ attributed to secondary and tertiary amines $\left(\mathrm{NH} / \mathrm{N}-(\mathrm{C})_{3}\right)$ ((Figure $\mathrm{S} 13$ and $\left.\mathrm{S} 14\right){ }^{[19]}$ An increase in the $\mathrm{NH} / \mathrm{N}-(\mathrm{C})_{3}$ peak was observed for the dodecyl-functionalised PTI compared to both FL-PTI and physisorption controls (Table S1). Quantitatively, the XPS data indicate 74 framework atoms per alkyl chain, comparing favourably with the TGA estimate of 101 atoms per chain. Controls of air quenched FL-PTI mixed with $\mathrm{C}_{12} \mathrm{H}_{25} \mathrm{Br}$ and to the unfunctionalised FL-PTI, precluding a contribution from physisorption. The XPS measurements also exclude physisorption since the core level $\mathrm{Br} 3 \mathrm{~d}$ was not observed in the grafted products, although it was visible in positive controls at relevant concentrations (Figure S15). Reaction with a shorter alkyl chain was also investigated; when charged FL-PTI reacted with octylbromide a mass loss of 12 wt $\%$ and the corresponding $\mathrm{m} / \mathrm{z}$ peak at 57 were observed (Figure S16). XPS showed a similar increase in the $\mathrm{NH} / \mathrm{N}$ $(C)_{3}$ peak as observed for the dodecyl-functionalised PTI (Figure S17 and Table S1). In this case, XPS indicates one alkyl chain every $47 \mathrm{PTI}$ atoms, closely matching the TGA estimate of one chain per 55 framework atoms. Given the uncertainties in these measurements, the agreement is excellent, and provides direct evidence of grafting to the PTI layers.

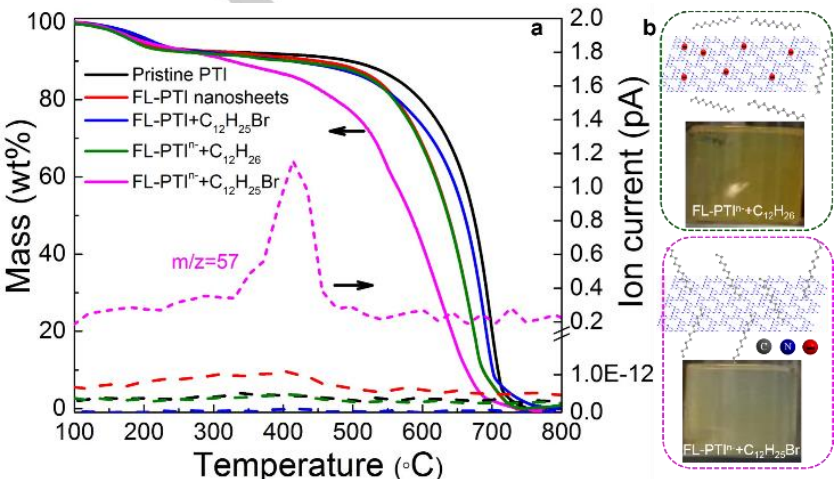

218 Figure 5. (a) TGA and TGA-MS (dashed) of pristine PTI and alkylated FL219 PTI. Inset shows $100-400^{\circ} \mathrm{C}$ TGA region. (b) Photographs of control 220 sample (top) and FL-PTI functionalised with dodecyl bromide (bottom).

221 The observed change in the XPS nitrogen components upon 222 functionalization can be attributed to the attachment of alkyl 223 chains to the nitrogen of the triazine ring. Density functional 224 theory (DFT) calculations suggest that the sodium ion 225 bridges two triazine rings, with the extra electron delocalized 226 over those two rings (Figure S19). The extra electron 227 appears to be primarily located on the nitrogen atoms of the 228 ring, which may therefore be most susceptible to react with 229 the alkyl bromide molecule (Scheme S2)

230 In summary, framework charging provides a new, simple 231 route for exfoliation and functionalisation of PTI nanosheets, 232 via NaNp/DMAc reduction. By avoiding damage, the intrinsic 233 properties of the PTI structure can be retained and HR 234 images indicate highly exfoliated hexagonal, crystalline FL235 PTI nanosheets, averaging $1.1 \mathrm{~nm}$ thick ( 3 layers) with $A B$ 236 stacking. The as-prepared FL-PTI solutions had a yield of 35 237 wt\%, with excellent stability

239 y. Stabilized dispersions of FL-PTIs are useful feedstocks for 240 a wide range of promising multifunctional applications. The 241 small flake size is particular relevant to potential applications 242 in (electro)catalysis and photochemistry. ${ }^{[20]}$ The FL-PTI ${ }^{\text {n- }}$ 243 was successfully functionalised with alkyl chains via the 
244 framework charge, suggesting a route to a wide range of 245 functionalised species to modulate surface chemistry and 246 functional properties.

\section{Acknowledgements}

248 Financial support within framework of the European Flagship 249 (grant agreement No. 696656-GrapheneCore1). CM 250 acknowledges the award of a Royal Society University Re251 search Fellowship by the UK Royal Society and the EPSRC 252 award EP/K033840/1. Work by TS, TS, VB, FC, CAH and 253 PFM at UCL was also supported by EPSRC grant 254 EP/L017091/1.

\section{Conflict of interest}

256 The authors declare no conflict of interest.

\section{References}

258

259 
[1] J. S. Zhang, Y. Chen, X. C. Wang, Energ Environ Sci 2015, 8, 3092-3108.

[2] M. Deifallah, P. F. McMillan, F. Cora, J Phys Chem C 2008, 112, 5447-5453.

[3] N. Mansor, T. S. Miller, I. Dedigama, A. B. Jorge, J. J. Jia, V. Brazdova, C. Mattevi, C. Gibbs, D. Hodgson, P. R. Shearing, C. A. Howard, F. Cora, M. Shaffer, D. J. L. Brett, P. F. McMillan, Electrochim Acta 2016, 222, 4457.

[4] G. G. Zhang, Z. A. Lan, X. C. Wang, Angew Chem Int Edit 2016, 55, 15712-15727.

[5] aP. Niu, L. L. Zhang, G. Liu, H. M. Cheng, Adv Funct Mater 2012, 22, 4763-4770; bS. B. Yang, Y. J. Gong, J. S. Zhang, L. Zhan, L. L. Ma, Z. Y. Fang, R. Vajtai, X. C. Wang, P. M. Ajayan, Adv Mater 2013, 25, 2452-2456; cT. Y. Ma, S. Dai, M. Jaroniec, S. Z. Qiao, Angew Chem Int Edit 2014, 53, 7281-7285; dM. J. Bojdys, N. Severin, J. P. Rabe, A. I. Cooper, A. Thomas, M. Antonietti, Macromol Rapid Comm 2013, 34, 850-854; eK. Schwinghammer, M. B. Mesch, V. Duppel, C. Ziegler, J. Senker, B. V. Lotsch, J Am Chem Soc 2014 , 136, 1730-1733.

[6] aE. Wirnhier, M. Doblinger, D. Gunzelmann, J. Senker, B. V. Lotsch, W. Schnick, Chem-Eur J 2011, 17, 32133221 ; bS. Y. Chong, J. T. A. Jones, Y. Z. Khimyak, A. I. Cooper, A. Thomas, M. Antonietti, M. J. Bojdys, J Mater Chem A 2013, 1, 1102-1107.

[7] K. Schwinghammer, B. Tuffy, M. B. Mesch, E. Wirnhier, C. Martineau, F. Taulelle, W. Schnick, J. Senker, B. V. Lotsch, Angew Chem Int Edit 2013, 52, 2435-2439.

[8] T. S. Miller, T. M. Suter, A. M. Telford, L. Picco, O. D. Payton, F. Russell-Pavier, P. L. Cullen, A. Sella, M. S. P. Shaffer, J. Nelson, V. Tileli, P. F. McMillan, C. A. Howard, Nano Lett 2017, 17, 5891-5896.

[9] J. M. Englert, C. Dotzer, G. A. Yang, M. Schmid, C. Papp, J. M. Gottfried, H. P. Steinruck, E. Spiecker, F. Hauke, A. Hirsch, Nat Chem 2011, 3, 279-286.

[10] aE. M. Milner, N. T. Skipper, C. A. Howard, M. S. P. Shaffer, D. J. Buckley, K. A. Rahnejat, P. L. Cullen, R. K. Heenan, P. Lindner, R. Schweins, J Am Chem Soc 2012, 134, 8302-8305; bP. L. Cullen, K. M. Cox, M. K. Bin Subhan, L. Picco, O. D. Payton, D. J. Buckley, T. S. Miller, S. A. Hodge, N. T. Skipper, V. Tileli, C. A. Howard, Nat Chem 2017, 9, 244-249.

[11] A. J. Clancy, J. Melbourne, M. S. P. Shaffer, J Mater Chem A 2015, 3, 16708-16715.

[12] N. G. Connelly, W. E. Geiger, Chem Rev 1996, 96, 877-910.

[13] S. H. A. Axdal, D. D. L. Chung, Carbon 1987, 25, 377389.

[14] aS. Fogden, C. A. Howard, R. K. Heenan, N. T. Skipper, M. S. P. Shaffer, Acs Nano 2012, 6, 54-62; bA. Penicaud, C. Drummond, Accounts Chem Res 2013 46, $129-137$.

[15] E. J. McDermott, E. Wirnhier, W. Schnick, K. S. Virdi, C. Scheu, Y. Kauffmann, W. D. Kaplan, E. Z. Kurmaev, A. Moewes, J Phys Chem C 2013, 117, 8806-8812.

[16] T. Morishita, A. J. Clancy, M. S. P. Shaffer, J Mater Chem A 2014, 2, 15022-15028.

[17] J. T. Yuan, J. J. Wu, W. J. Hardy, P. Loya, M. Lou, Y. C. Yang, S. Najmaei, M. L. Jiang, F. Qin, K. Keyshar, H. Ji, W. L. Gao, J. M. Bao, J. Kono, D. Natelson, P. M. Ajayan, J. Lou, Adv Mater 2015, 27, 5605-5609.

[18] H. Shin, J. W. Guan, M. Z. Zgierski, K. S. Kim, C. T. Kingston, B. Simard, Acs Nano 2015, 9, 12573-12582.

[19] D. Mitoraj, H. Kisch, Angew Chem Int Edit 2008, 47, 9975-9978.

[20] aN. Mansor, J. J. Jia, T. S. Miller, T. Suter, A. B. Jorge, C. Gibbs, P. Shearing, P. F. McMillan, C. Mattevi, M. Shaffer, D. J. L. Brett, Ecs Transactions 2016, 75, 885- 
Killing two birds with one stone: Single and few-layered 2D intact crystalline carbon nitride nanosheets in an $A B$ stacking structure were synthesized by a scalable one-pot reductive framework charging process. The novel negatively charged salt shows excellent stability under nitrogen and can be rationally functionalized by alkylation to tailor the properties of carbon nitride.

Jingjing Jia, Edward R. White, Adam J. Clancy, Noelia Rubio, Theo Suter, Thomas S. Miller, Paul F. McMillan, Veronika Brázdová, Furio Corà, Chris A. Howard, Robert V. Law, Cecilia Mattevi, ${ }^{*}$ and Milo S. P. Shaffer*

\section{Page No - Page No}

Fast Exfoliation and functionalization of 2D Crystalline Carbon Nitride by Framework Charging 